Thorax_(1971), 26, 638.

\title{
Aneurysms of the thoracic aorta
}

\author{
B. T. LE ROUX, M. A. ROGERS, and M. S. GOTSMAN \\ Thoracic and Cardiac Units, Wentworth Hospital, and the Departments of Surgery and Medicine, \\ University of Natal
}

\begin{abstract}
Selected radiographs from 40 patients with thoracic aortic aneurysm serve to illustrate most of the radiographic features of this disease. Surgical techniques are outlined and were used to modify the natural course of the disease in 14 patients, with three postoperative deaths. The remaining 26 patients were either moribund on admission and died shortly afterwards or declined operations and died later.
\end{abstract}

The purpose of this paper is to review the natural history, surgical management, and clinical and radiographic features of aneurysm of the aorta above the diaphragm. Experience is limited to 40 patients, of whom only nine were women. An operation, the aim of which was to resect the aneurysm, was undertaken in 14 patients; three of these died in the early postoperative period and the others are well. All of the 26 patients in whom an operation was not undertaken have died. The youngest patients were men of 27 and 33 years, respectively, in whom the diagnosis of cystic medial necrosis in association with Marfan's syndrome was confirmed post mortem : five were in the fourth decade, 10 in the fifth, 10 in the sixth, 12 in the seventh, and the two oldest were 70 and 71 years respectively. Most (33) were Bantu and in them age was often an estimate; four were Asiatic and three of Caucasian stock. There was serological evidence of luetic disease in 27 ; in eight, in three of whom the Wassermann reaction was positive, there was a history of trauma of such severity that it would not have been surprising if the aneurysm had had a traumatic origin, and in four of these traumatic aneurysm was diagnosed with certainty at thoracotomy; in 15 patients, in four of whom the Wassermann reaction was positive, the aneurysm was associated with extensive and severe atherosclerosis of the aorta. The aneurysm was distal to the left subclavian artery in 16 patients-well distal in 10, and barely distal in six. In six, the aneurysm involved the arch ; in seven, the ascending aorta, short of the innominate artery; in seven, the innominate artery was aneurysmal-in five of these with aneurysmal dilatation of the ascending aorta; in five of the last-mentioned 14 patients there was aortic incompetence. In three patients, two with cystic medial necrosis, the entire thoracic aorta was aneurysmal, and in the last there were several small aneurysms of the ascending aorta in association with aortic stenosis.

\section{PRESENTATION}

A painful pulsatile swelling of the chest wall prompted admission to hospital in five patients; in four the swelling was sternal (Fig. 1) and in one it was extensive and included the left posterior chest wall (Fig. 2). Pain was a prominent symptom in 15 other patients, in all of whom there was radiographic evidence of vertebral erosion. One of these patients was admitted in extremis with paraplegia, and destruction of the spinal cord at mid-thoracic level was confirmed post mortem. Dysphagia was a symptom in nine patients, was total in three, was the symptom which prompted admission in these and in three others, and was incidental in three. In none was dysphagia the only symptom. Hoarseness of voice was the only complaint in four patients, and was associated with other symptoms in eight. The clinical features of obstruction of the superior vena cava, present for a month, became rapidly more incapacitating and were the reason for seeking admission in one patient. In him, venous distention of the neck was pulsatile and necropsy revealed a fistula between an aneurysm of the ascending aorta and the superior vena cava. In three other patients obstruction of the superior vena cava was incidental. Two patients were admitted urgently dyspnoeic and died from suffocation consequent upon tracheal obstruction. Stridor was a feature in five other patients. Dyspnoea was one of several complaints 


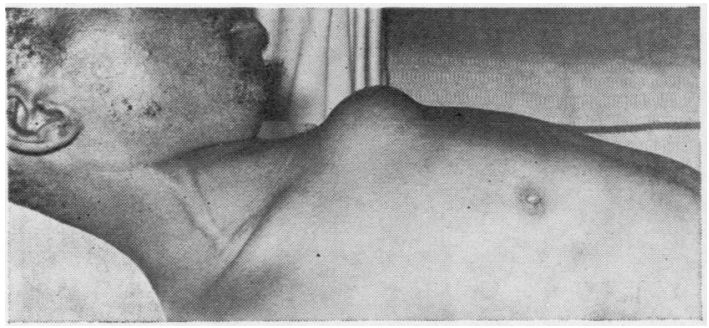

FIG. 1. Sternal erosion from thoracic aortic aneurysm.
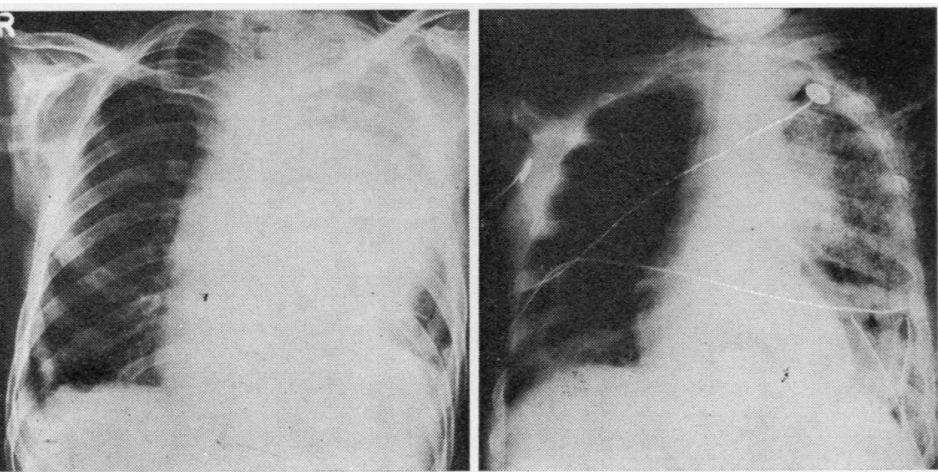

FIG. 3. Pre- and post-operative anteroposterior chest radiographs of the patient shown in Fig. 2. There is erosion of the 8th, 9th, 10th, and 11 th ribs, including the posterior rib angle. Management included left lower lobectomy and replacement of the descending thoracic aorta with tubular Dacron.
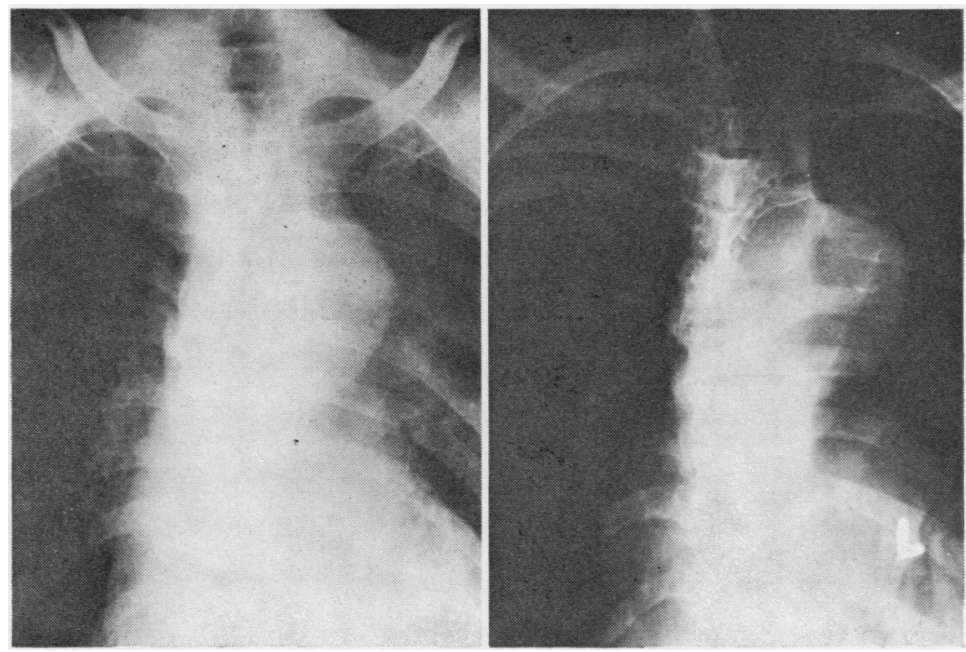

FIG. 4. Vertebral erosion from descending thoracic aortic aneurysm, with paraplegia. interval close to the angle of the left scapula.

FIG. 2. Erosion of left lateral and posterior chest wall with presentation of a descending thoracic aortic aneurysm as a pulsating haematoma, pointing in the scapulovertebral

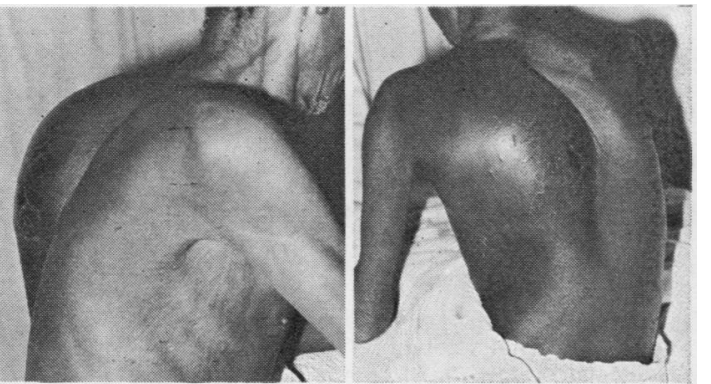



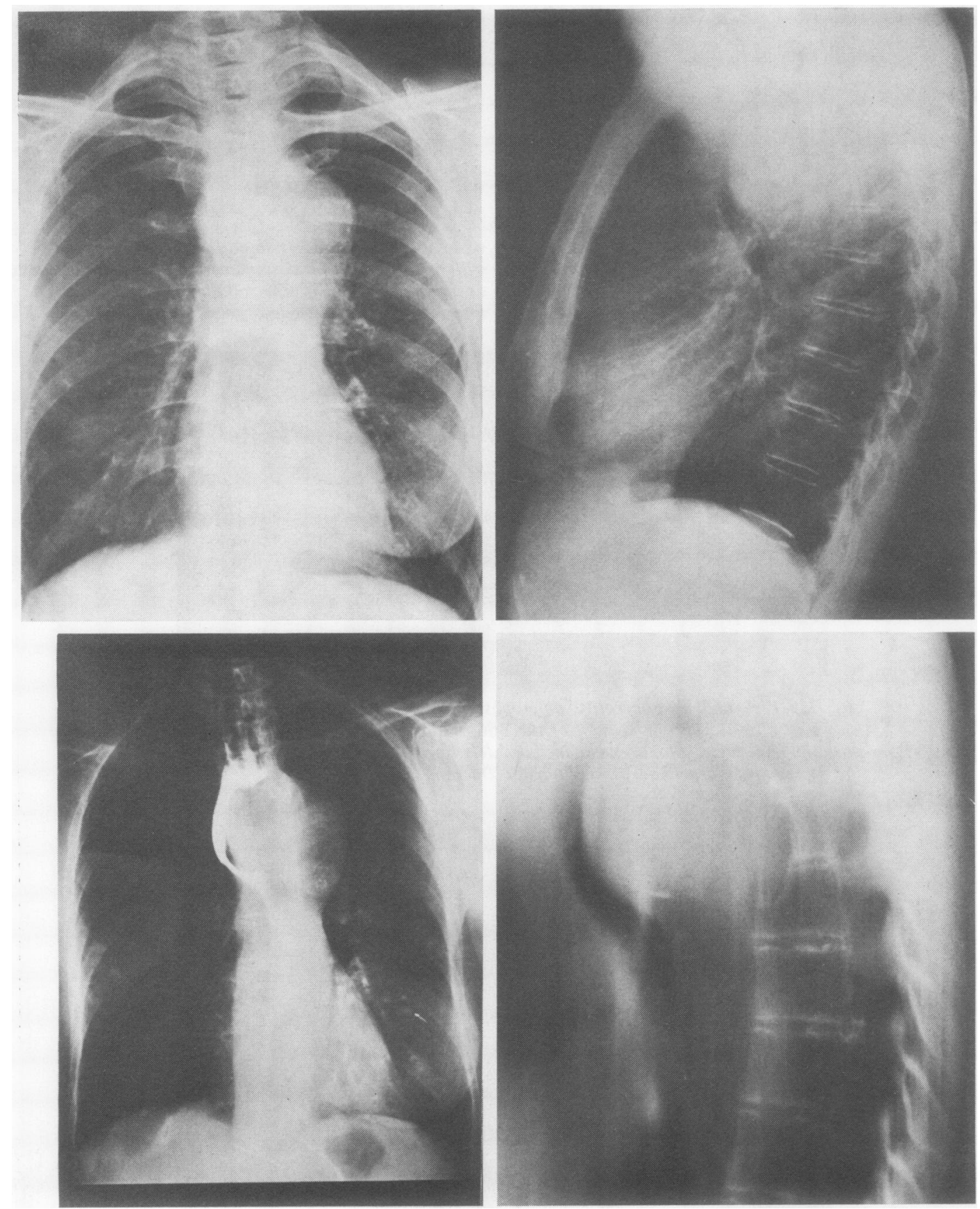

FIG. 5. An aneurysm of the descending thoracic aorta in a patient previously submitted to right mastectomy for carcinoma, who presented with hoarseness of voice and stridor. Radiographically there is calcification in the aneurysm, tracheal and oesophageal displacement, and vertebral erosion. 
in two patients shown to have obstructive emphysema of the left lung related to compression of the left stem bronchus. Ingravescent haemoptysis in five patients prompted admission, and death in four was from haemorrhage related to direct communication between an aneurysm and the lung with erosion of a major bronchus.

\section{RADIOGRAPHIC APPEARANCES}

Most of the reported radiographic features of thoracic aortic aneurysm were found in this group of 40 patients. Bones eroded included the sternum, ribs (Fig. 3), and vertebrae (Figs 4 and 5). Tracheal compression and displacement were more constantly recognized on lateral films and tomo- graphically (Figs 5, 6, and 7). Oesophageal compression and displacement were demonstrated with barium at fluoroscopy and recorded (Figs 5 and 8). Phrenic nerve interruption, suspected from the height of a diaphragmatic dome, was confirmed fluoroscopically (Fig. 9). Obstructive emphysema, clearly evident radiographically (Fig. 10), was associated with obstruction of the left main bronchus, seen at bronchoscopy. Calcification of the aneurysm was common (Figs 5, 10, and 11). More than half the patients with thoracic aortic aneurysms were referred with the provisional diagnosis of bronchial carcinoma. The configuration of the opacity made this a reasonable diagnosis in some (Fig. 9), and association with superior vena caval obstruction in four patients, until this was recog-

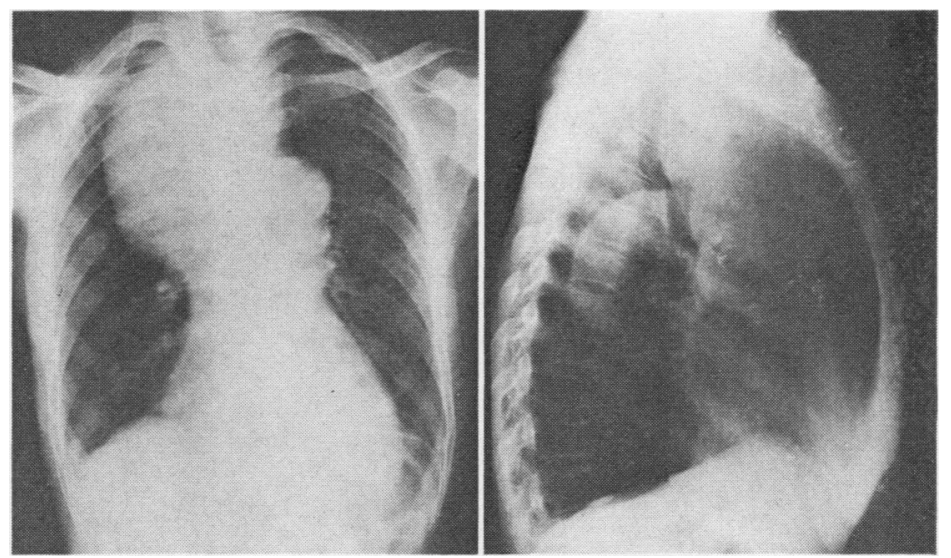

FIG. 6. Aneurysm of the ascending aorta in a patient who presented because of stridor and died shortly after admission from suffocation. Posterior displacement of the trachea is well seen.

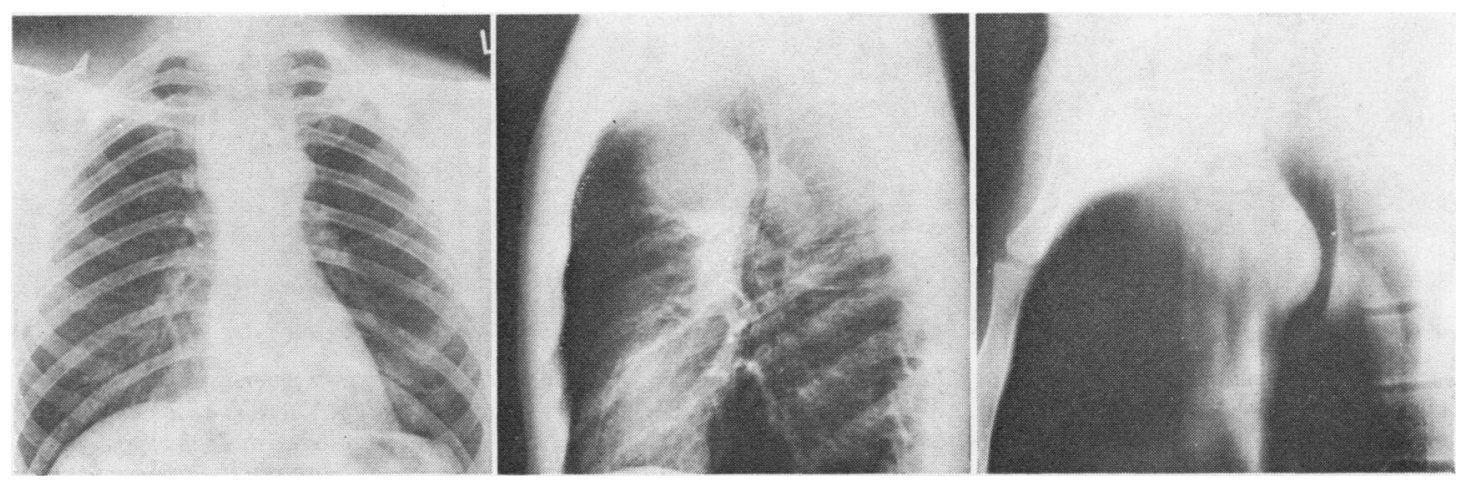

FIG. 7. Innominate aneurysm associated with tracheal compression. 

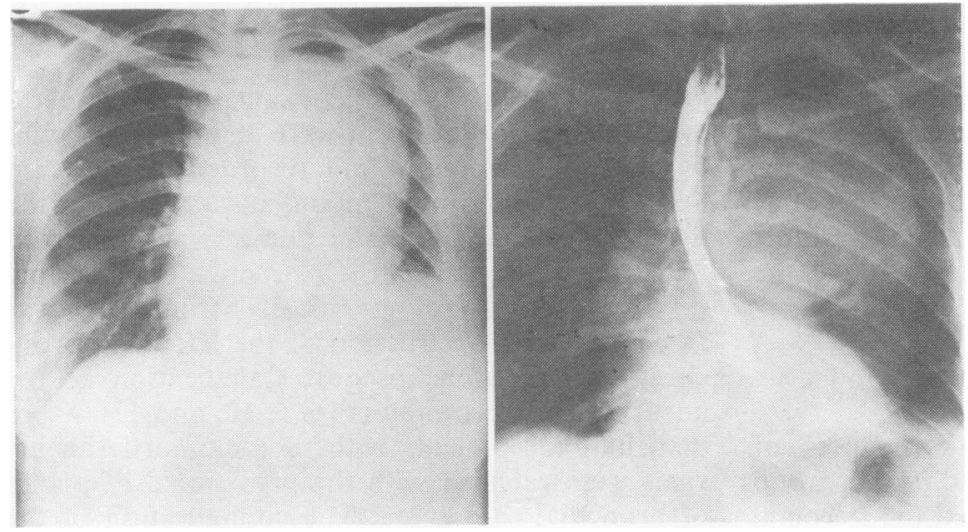

FIG. 8. An aneurysm which involved much of the arch and the descending thoracic aorta associated with tracheal and oesophageal displacement and displacement and compression of the left stem bronchus.

FIG. 9. Aneurysm of the ascending aorta associated with phrenic paresis. This patient was referred for investigation with the diagnosis of bronchial carcinoma.
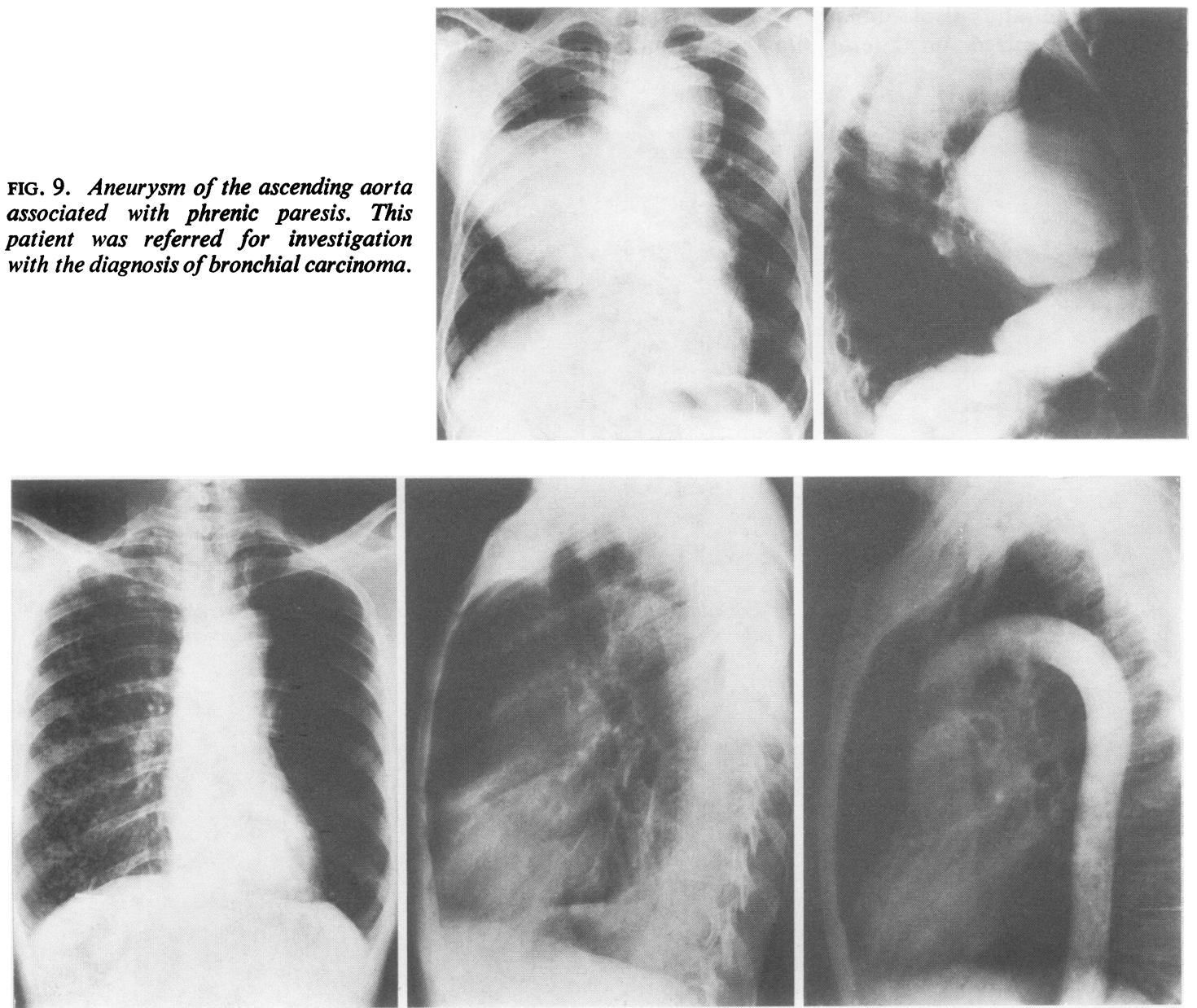

FIG. 10. Thoracic aortic aneurysm, saccular in type, originating at the site of the ligamentum arteriosum and occupying the aortic window. Calcification can be seen in relation to the lower border of the aortic arch. There is obstructive emphysema of the left lung. Presentation was with hoarseness of voice and dyspnoea. The aortogram is normal; the space between the ascending and descending limbs may be wider than normal. At thoracotomy, with the provisional diagnosis of thoracic aortic aneurysm, the aneurysm was seen to be saccular, the size of a small orange, and completely occupied by clot. Obstruction of the left stem bronchus was relieved by resection of the aneurysm. 


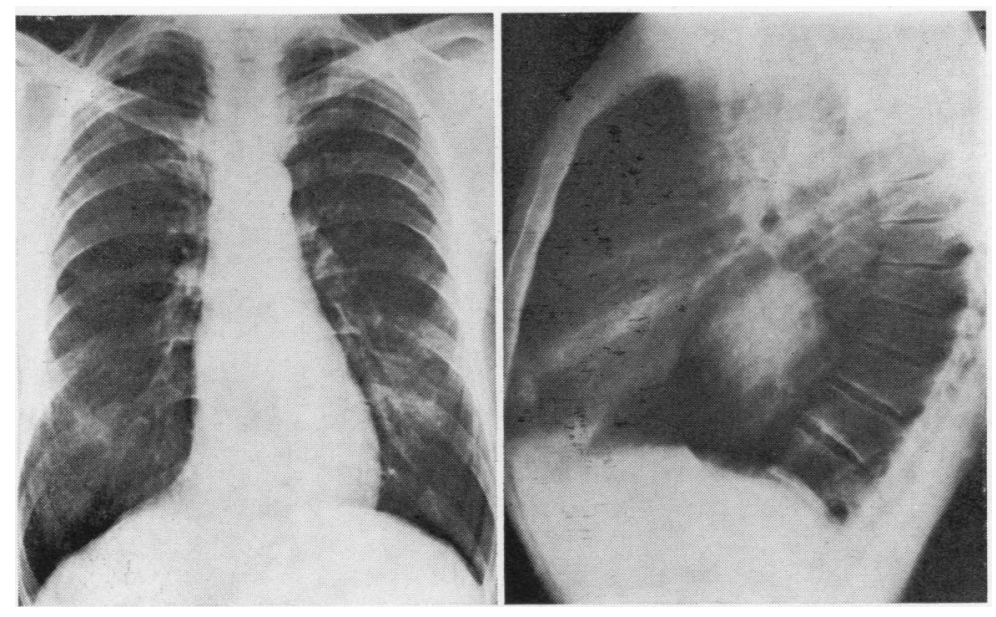

FIG. 11. Aneurysm of the descending thoracic"aorta with calcification of the wall. Presentation was with dysphagia.

FIG. 12. Aneurysm of the ascending aorta with fistulous communication with the superior vena cava.
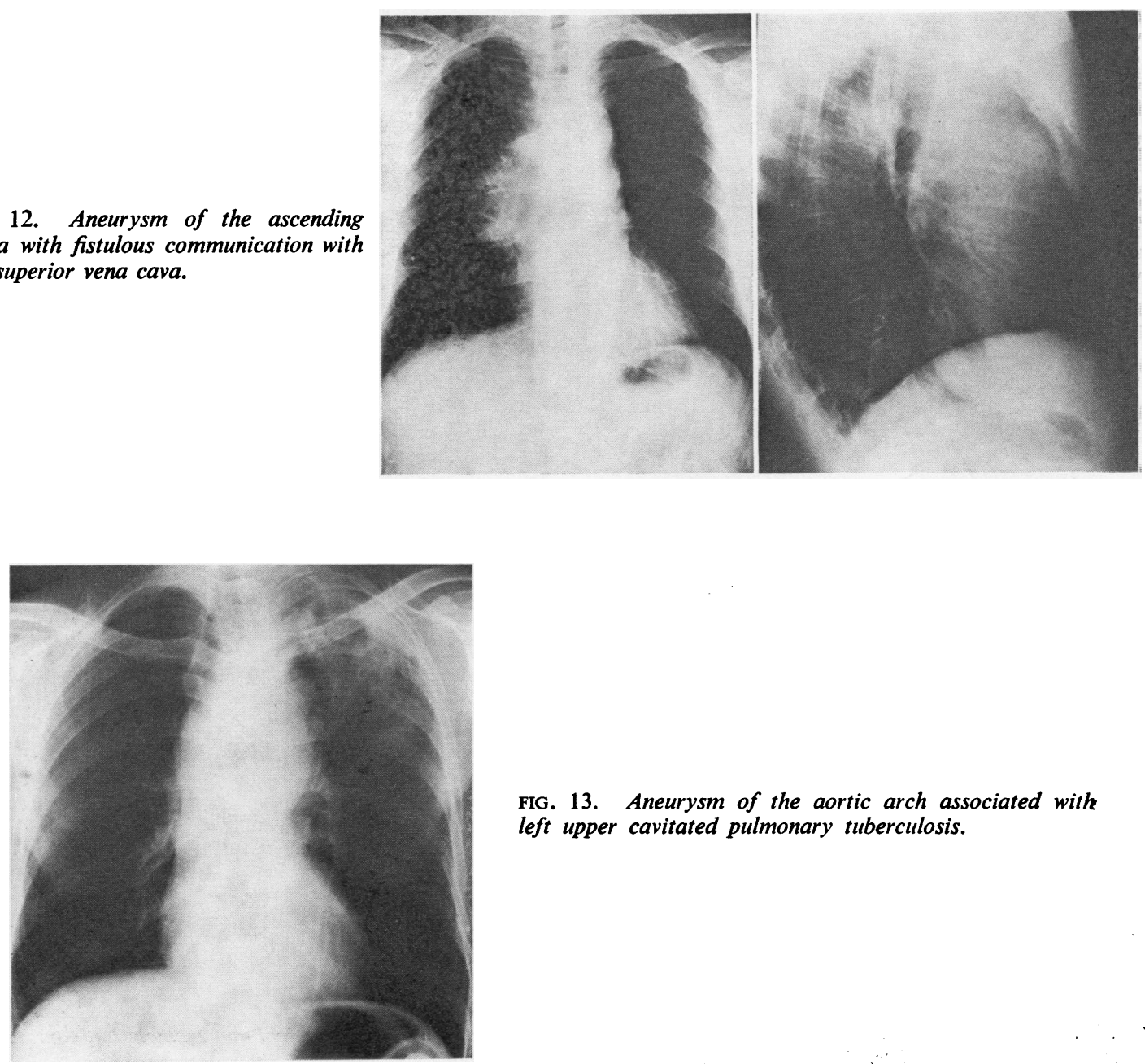

FIG. 13. Aneurysm of the aortic arch associated with left upper cavitated pulmonary tuberculosis. 

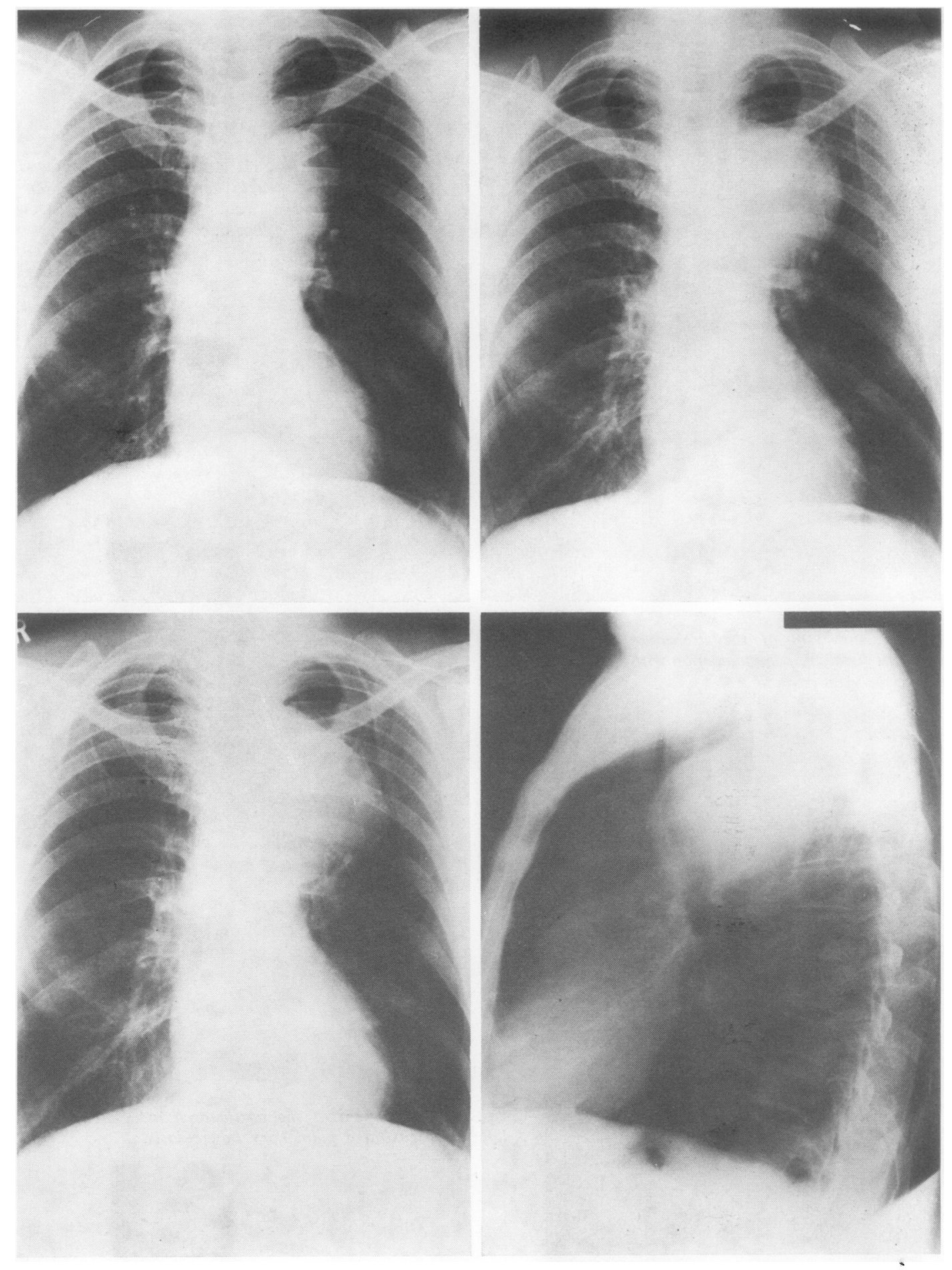

FIG. 14. Growth of the aortic arch aneurysm over 18 months. This patient declined surgical management but continued to attend regularly for review. Death was from torrential haemorrhage. 
nized as pulsatile in one (Fig. 12), made bronchial carcinoma a reasonable alternative diagnosis. Associated lesions recognized radiographically included cavitated pulmonary tuberculosis (Fig. 13) in three patients in whose sputum tubercle bacilli were identified. Reluctance on the part of a number of patients to submit to surgical man- agement-some even to investigation-offered the opportunity of radiographic demonstration of growth of thoracic aortic aneurysm (Figs 14 and 15). Aneurysm of the ascending aorta usually prolapses to the right and obscures the right heart border on postero-anterior films, because both are predominantly anterior. Aneurysm of the descend-
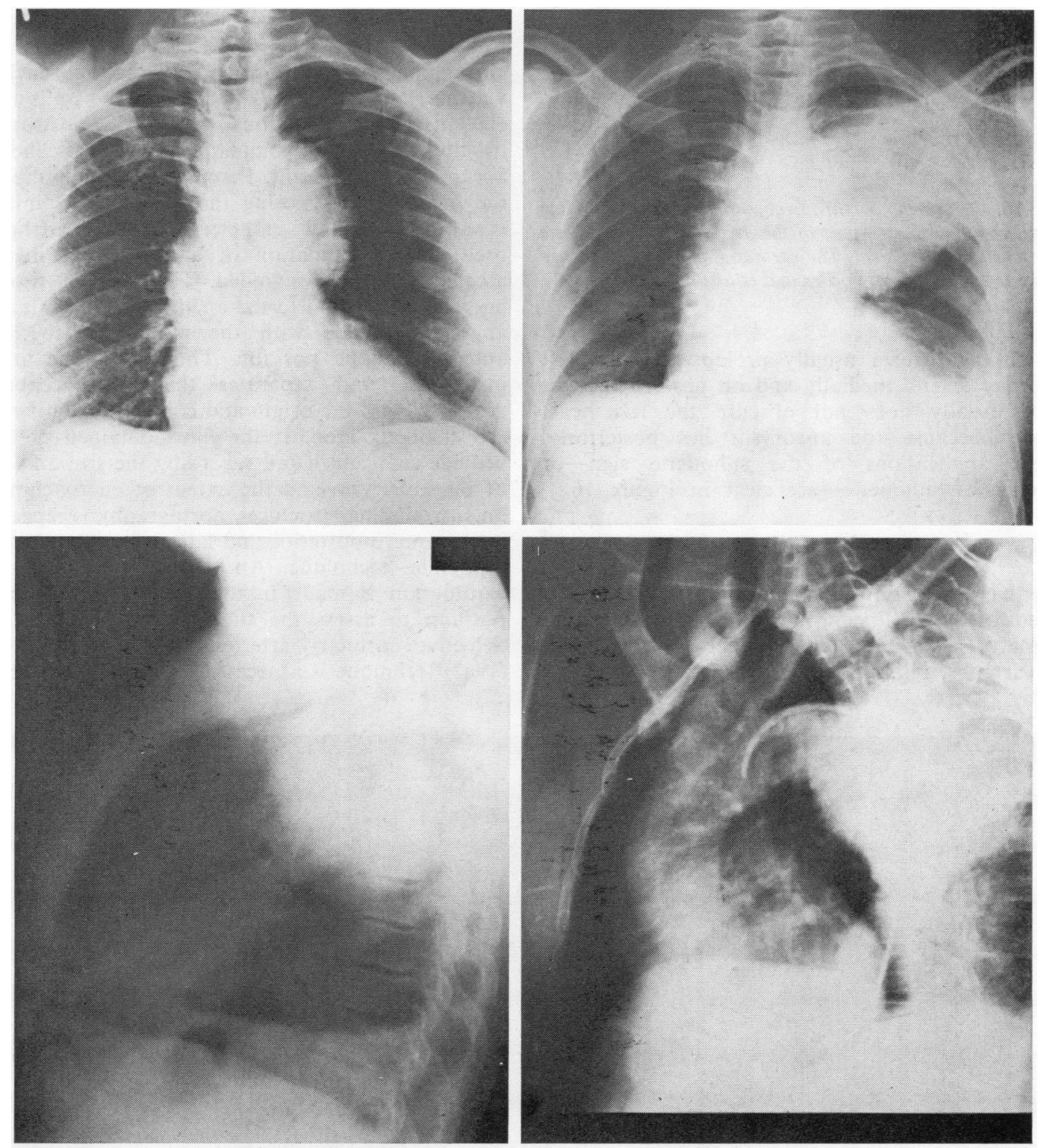

FIG. 15. Descending thoracic aortic aneurysm showing growth over six months. The size of the aneurysm demonstrated angiographically is nearly commensurate with the size demonstrated radiographically. 


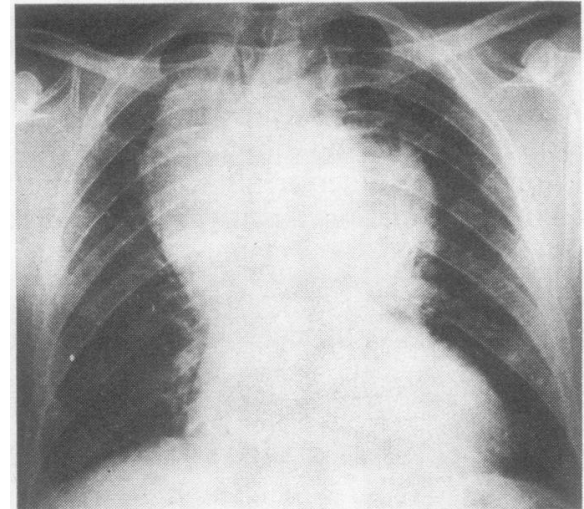

FIG. 16. Thoracic aortic aneurysm involving ascending aorta, aortic arch, and part of the descending aorta. There is tracheal displacement. The cardiac silhouette is retained at the left mediastinal border and is lost on the right.

ing thoracic aorta usually prolapses to the left but may extend medially and on postero-anterior films usually does not obscure the left heart border because the aneurysm lies posteriorly. These applications of the silhouette sign-or absence of silhouette-are clear in Figure 16.

\section{ANGIOGRAPHY}

This has as its aim demonstration of the site and extent of the aneurysm, the degree of involvement of the vessels to the head and neck and the coronary arteries, relationship of the aneurysm to the surrounding structures, and the state of the aortic valve, and serves to confirm the diagnosis in most instances.

Selective aortography, by injection of contrast medium directly into the area under study, is safe, and there is not the need for prograde techniques which depend on bilateral peripheral venous or direct pulmonary arterial injections. Serial radiography with $14 \times 14$ inch cut films at a rate of 3 to 6 per second demonstrates well the anatomical abnormality and is a technique superior to cineangiography. The aorta can be approached via the right brachial artery, but if the right subclavian artery is tortuous or partially obstructed by the aneurysm, cannulation of the ascending aorta may be difficult. Percutaneous puncture of the femoral artery using the Seldinger technique is safe if a 'pigtail ' catheter with a curved tip is used and manipulation of a guide wire in the ascending aorta is avoided. The catheter should be placed proximal to the aneurysm and an initial angiogram made with the patient in the left anterior oblique position. This places the aorta in profile and separates the brachiocephalic vessels so that the origin and course of each vessel are distinctly seen. If the films obtained do not provide sufficient detail regarding the size and site of the aneurysm and the extent of encroachment on surrounding structures, aortography is repeated in the antero-posterior and lateral positions, using a biplane technique. An aortic root cineangiocardiogram is made in the left anterior oblique position to assess the state of the aortic valve. Selective coronary arteriography using Judkins' (1967) technique is a necessary part of the investi-
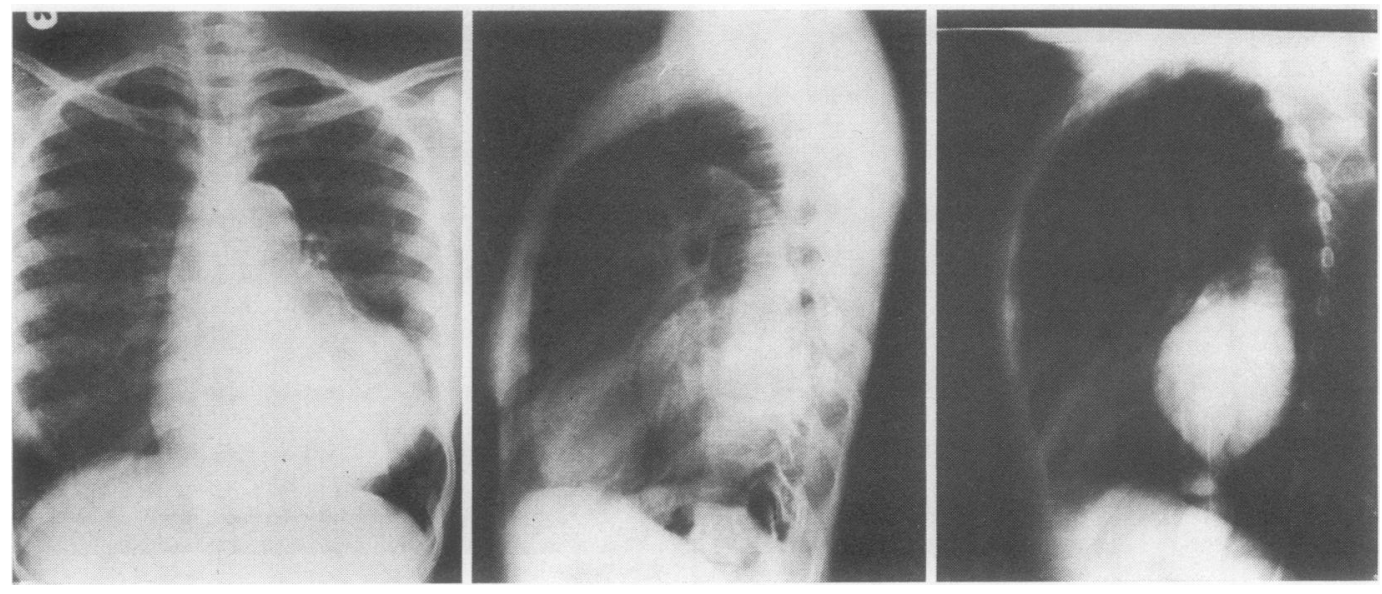

FIG. 17. Aneurysm of the descending thoracic aorta in which there is a considerable layer of clot. 
gation but may be technically difficult if the aneurysm involves the root of the aorta. Coronary ostial involvement is not uncommon in syphilitic aortitis.
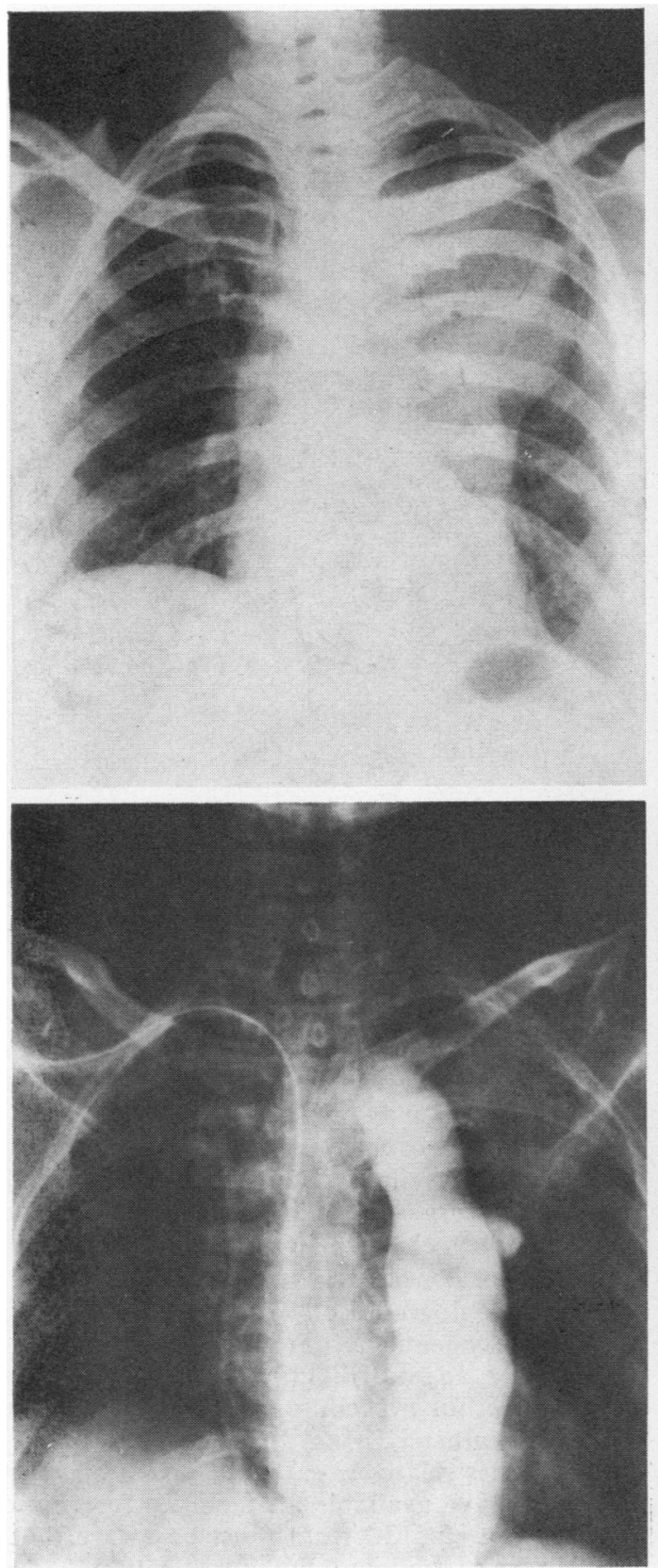

While it is well recognized that the angiographic demonstration of the size of the aortic aneurysm is not necessarily commensurate with the actual size of the aneurysm, because the lumen
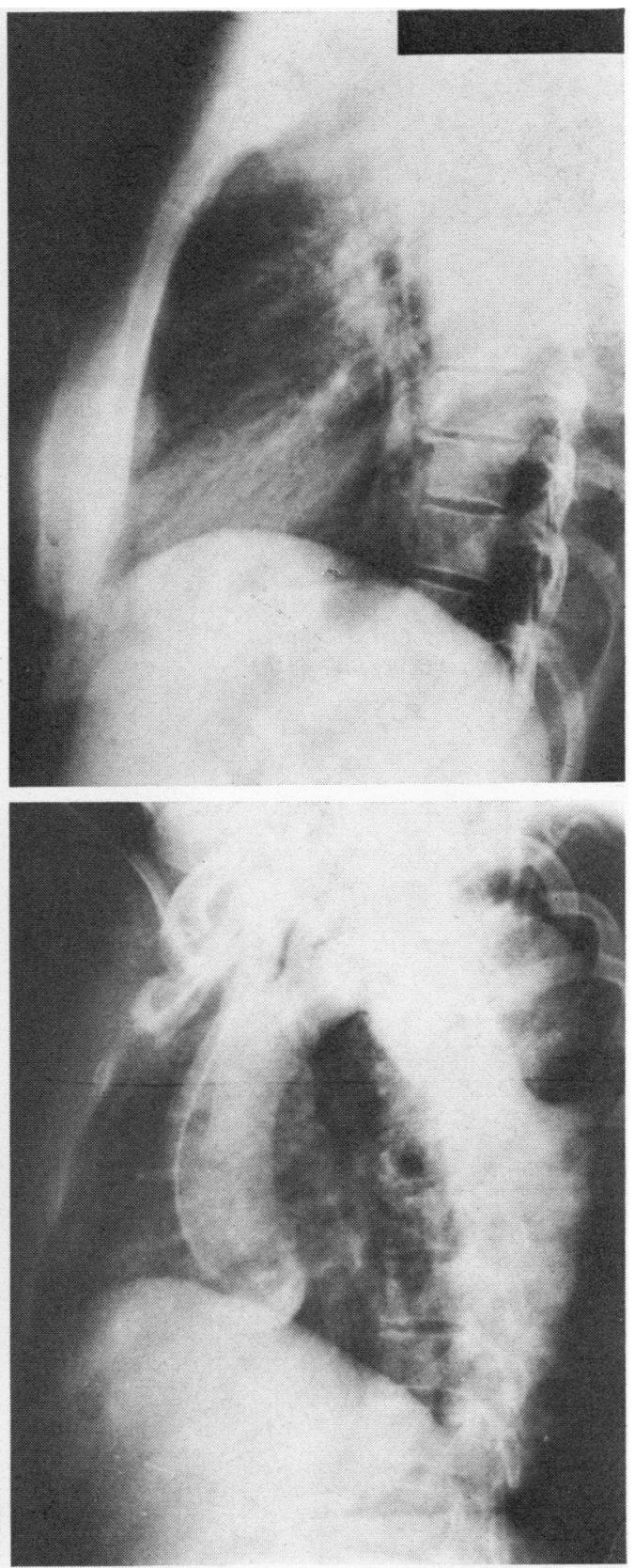

FG. 18. Aneurysm of the descending thoracic aorta in which the size of aortic lumen demonstrated angiographically is not commensurate with the size of the aneurysm because of the large volume of contained clot. 


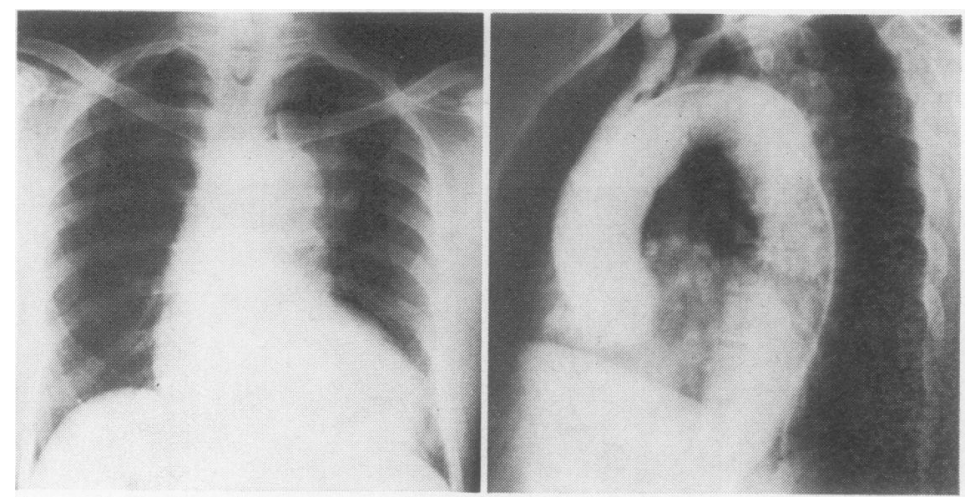

FIG. 19. Aneurysm of the descending thoracic aorta immediately distal to the left subclavian artery with a nearly normal aortogram.

FIG. 20. A remarkably tortuous aorta associated with an aneurysm of the innominate artery.

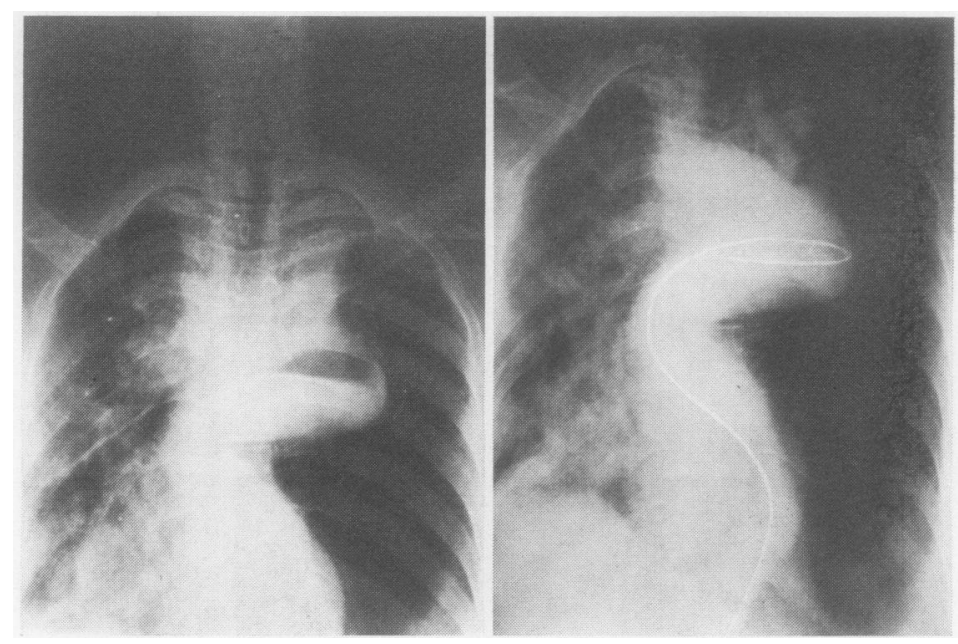

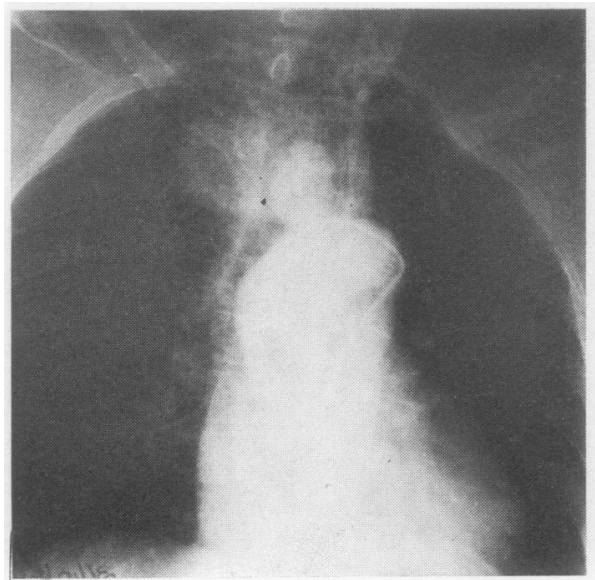

Fo. 21. Innominate artery aneurysm containing a large volume of clot. of the aneurysm is more or less occupied by clot (Figs 17 to 21), it is less generally realized that the aortogram may in fact be normal (Fig. 10). Failure to fill coronary arteries with contrast may make operative intervention pointless (Fig. 22) to manage surgically one facet of degenerative or inflammatory changes in the cardiovascular system if there is evidence both electrocardiographically and angiographically that the myocardium is severely ischaemic is unrewarding.

Selective angiocardiography has been regarded as a dangerous procedure. If undertaken skilfully, with the catheter in an appropriate position, the procedure is relatively safe, but provision must be made to have available all facilities for resuscitation, and a surgical team must be aware that the patient is to undergo the investigation and that an emergency thoracotomy may be necessary. Respiratory obstruction from an aneurysm is 

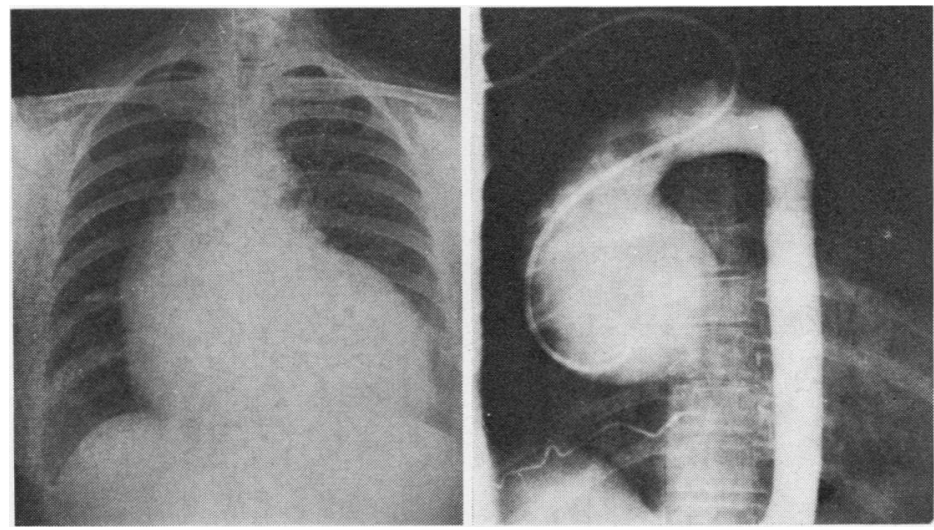

FIG. 22. Aneurysm of the ascending aorta without aortic incompetence. Coronary arteries are not filled. This is not unusual in relation to an injection of contrast into the aorta and selective coronary angiography is required to determine accurately whether or not the coronary arteries are obstructed.

occasionally aggravated by aortography, and in these circumstances an anaesthetist must be available should it become necessary to intubate the trachea.

\section{MANAGEMENT}

In the absence of an acceptable alternative form of management, all patients with thoracic aortic aneurysms considered likely to survive an operation were offered surgical treatment. Seven patients were regarded as unsuitable for any form of treatment, so close to death were they at the time of admission. All had died within 48 hours. Five patients died during the course of investigation; there is no evidence that investigation precipitated death: death in four was from torrential haemorrhage and in one, with a fistulous communication between the aneurysm and the superior vena cava, was abrupt and probably from myocardial infarction. An operation was undertaken in 14 patients, and offered as the only effective management to the remaining 14 patients, who declined, although some continued to attend regularly until they died.

Surgical techniques adopted depended on the site of the aneurysm. Those aneurysms limited to the ascending aorta were managed with total cardiopulmonary bypass and with left coronary artery perfusion. More extensive aneurysms, which included the great branches of the arch, were managed similarly and, in addition, the right carotid arteries were perfused through the right axillary artery and the left carotid arteries either by cannulation of the left common carotid artery through its wall, or through its lumen from within the aneurysm with a Foley's catheter. Aneurysms of the descending thoracic aorta were managed with partial cardiopulmonary bypass, by cannulation of the left femoral artery and vein, and direction of blood from vein to artery through a bubble oxygenator, the volume of flow being determined by the need to maintain rostral blood pressure at normal or slightly elevated levels. The aortic valve was replaced in two patients in conjunction with replacement of the ascending aorta. Aortic replacement was with crimped, tubular, woven Dacron. Sternal erosion (Fig. 1) did not make unusually difficult management of two patients whose aneurysm presented through the sternum and which involved, in one, only the ascending aorta (Fig. 23) and in the other most of the arch. The patient whose aneurysm presented through the left posterolateral chest wall (Fig. 2) was shown to have a descending thoracic aortic aneurysm, but so vast was the cavity of the aneurysm, both true and false, that contrast studies were of insufficient clarity to justify reproduction. Management included left lower lobectomy since the lobe was incorporated in the wall of a false aneurysm. Most of four ribs were destroyed posterolaterally, and there was vertebral erosion. The aneurysm had an origin which was quite short-about $8 \mathrm{~cm}$ in length-from the descending thoracic aorta and which began some $4 \mathrm{~cm}$ distal to the left subclavian artery. The sac had eroded the posterolateral chest wall and the left lower lobe, and then ruptured into the soft tissues of the back. Attenuation of the integument 


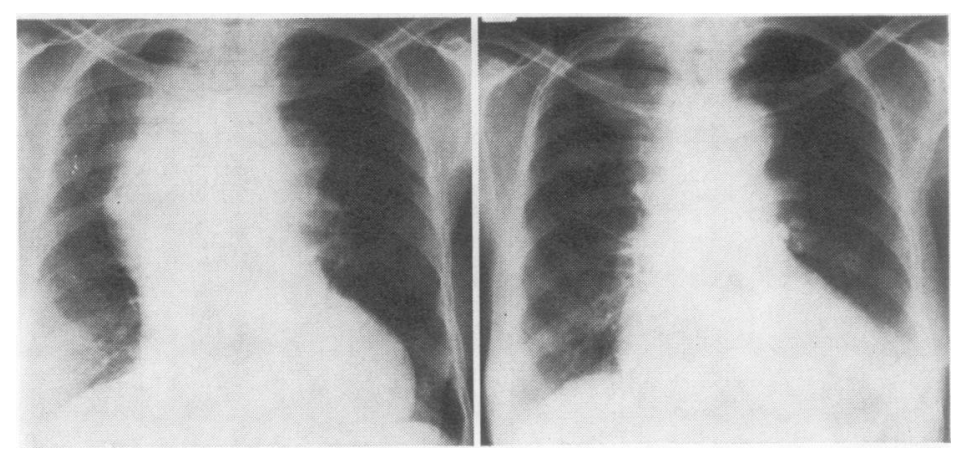

FIG. 23. This patient presented with a pulsatile swelling of the sternum. The aneurysm was saccular and involved the ascending aorta. Management was by resection and replacement of the ascending aorta with tubular Dacron. Preoperative and early postoperative radiographs.

of the back is clear from the clinical photographs.

There were three deaths in relation to operation: one patient failed to recover consciousness and is presumed to have had cerebral embolism, of either air or clot; one patient died on the evening of operation from myocardial infarction, a diagnosis confirmed at necropsy; the third patient died suddenly on the eighteenth postoperative day, having been well in every respect and ambulant, and death may have been from pulmonary embolism, but necropsy evidence of this could not be obtained through lack of consent to the examination.

\section{DISCUSSION}

Among the Bantu population of the Province of Natal in South Africa there persists a large measure of indifference to personal discomfort, distrust of conventional western medicine, and faith in the pronouncement of witchdoctors. Oesophageal carcinoma, for example, is operable in fewer than $2 \%$ of patients with this disease, and most attend hospital with total dysphagia, oesophagotracheal fistula, and extensive pulmonary damage from aspiration (Levy, 1966 ; Procter, 1968). Even such an obvious embarrassment as a fungating chest-wall tumour is tolerated long beyond any acceptable western standards and long after it has become impossible to wear conventional clothing (le Roux and Stemmler, 1971). It is, therefore, not surprising that clinical experience with thoracic aortic aneurysm in this population group should be similar to that in western countries before the advent of techniques in vascular surgery which has so greatly modified the natural history of the disease (Keefer and Mallory, 1934). The surgical techniques employed have been well described (Bloodwell, Hallman, and Cooley, 1966 ; Bryant, Bowlin, Malette, and Danielson, 1967). The techniques of maintenance of a distal circulation by some form of partial bypass during management of aneurysms distal to the left subclavian artery are possibly unnecessary (Crawford, Fenstermacher, Richardson, and Sandiford, 1970). There is unequivocal evidence that successful surgical management of thoracic aortic aneurysms modifies favourably the natural history of the disease (De Bakey, Cooley, and Creech, 1957).

Since an early report on the complications of aortography (Crawford, Beall, Moyer, and De Bakey, 1957) techniques have been modified and contrast media improved, so that the mortality and morbidity of this investigation are now small, but in patients in whom thoracic aortic aneurysm is far advanced, death from haemorrhage in close relation to investigation may be significant and not merely evidence of the inexorable advance of the disease, unmodified by investigation.

Many of the aneurysms reported in this series were so large that distinction between fusiform and saccular varieties was a matter of opinion. Some were undoubtedly saccular but none was suitable for management by the application of a lateral clamp, amputation of the aneurysm, and lateral repair of the aorta. None was dissecting in type. 
Some of the illustrations (Figs 5, 10, 11, and 22) have been published previously in $A$ Portfolio of Chest Radiographs and $A$ Second Portfolio of Chest Radiographs (Le Roux and Dodds, 1964 and 1968 ; Livingstone, London and Edinburgh). Three illustrations (Figs 5, 11, and 22) do not relate to any of the 40 patients reported in this series; they are included as they serve to illustrate a particular radiographic feature better than similar radiographs of poor quality from the series reported. The patients to whom these three illustrations relate were managed by Mr. Andrew Logan in the Thoracic Unit, Royal Infirmary, Edinburgh, whose permission to use them in this context is gratefully acknowledged.

\section{REFERENCES}

Bloodwell, R. D., Hallman, G. L., and Cooley, D. A. (1966). Aneurysm of the ascending aorta with aortic valvular insufficiency: surgical management. Arch. Surg., 92, 588.
Bryant, L. R., Bowlin, J., Malette, W., and Danielson, G. K. (1967). Thoracic aneurysms with aortico-bronchial fistula. Ann. Surg., 168, 79.

Crawford, E. S., Beall, A. C., Moyer, J. H., and De Bakey, M. E. (1957). Complications of aortography. Surg. Gynaec. Obstet., 104, 129.

- Fenstermacher, J. M., Richardson, W., and Sandiford, F. (1970). Reappraisal of adjuncts to avoid ischemia in the treatment of thoracic aortic aneurysms. Surgery, 67, 182.

De Bakey, M. E., Cooley, D. A., and Creech, O. (1957). Aneurysm of the aorta treated by resection. Analysis of 313 cases. J. Amer. med. Ass., 163, 1439.

Judkins, M. P. (1967). Selective coronary arteriography. Part I: A percutaneous transfemoral technic. Radiology, 89, 815.

Keefer, C. S., and Mallory, G. K. (1934). The pulmonary and pleural complications of aortic aneurysm. Amer. Heart J., 10, 208.

le Roux, B. T., and Stemmler, P. (1971). Maintenance of chest wall stability: a further report. Thorax, 26, 424.

Levy, J. I. (1966). The diagnosis of carcinoma of the eosophagus in the plain chest radiograph. $S$. Afr. J. Radiol., 4, 1.

Procter, D. S. C. (1968). Carcinoma of the oesophagus. A review of 523 cases. S. Aftr. J. Surg., 6, 137. 